\title{
Effect of scheme transformations on a beta function with vanishing one-loop term
}

\author{
Thomas A. Ryttov $\oplus^{1}$ and Robert Shrock $\oplus^{2}$ \\ ${ }^{1} C P^{3}$-Origins, Southern Denmark University, Campusvej 55, Odense, Denmark \\ ${ }^{2}$ C. N. Yang Institute for Theoretical Physics and Department of Physics and Astronomy, \\ Stony Brook University, Stony Brook, New York 11794, USA
}

(Received 15 August 2020; accepted 21 August 2020; published 15 September 2020)

\begin{abstract}
It is commonly stated that because terms in the beta function of a theory at the level of $\ell \geq 3$ loops and higher are scheme dependent, it is possible to define scheme transformations that can be used to remove these terms, at least in the vicinity of zero coupling. We prove that this is not, in general, possible in the situation where a beta function is not identically zero but has a vanishing one-loop term.
\end{abstract}

DOI: 10.1103/PhysRevD.102.056016

\section{INTRODUCTION}

Let us consider a quantum field theory in $d$ spacetime dimensions, with some set of fields and a dimensionless interaction coupling $g$. Two cases of particular interest are a non-Abelian gauge theory in $d=4$ dimensions, where $g$ is the gauge coupling, and a scalar theory with a cubic interaction in $d=6$ dimensions, where $g$ is the coefficient of the cubic interaction. Quantum corrections render $g$ dependent upon the Euclidean energy/momentum scale $\mu$ where it is measured: $g=g(\mu)$. The dependence of $g$ on $\mu$ is determined by the renormalization-group (RG) beta function of the theory, $\beta_{g}=d g / d t$, where $d t=d \ln \mu$ [1]. This function has the series expansion

$$
\beta_{g}=g \sum_{\ell=1}^{\infty} b_{\ell} a^{\ell}
$$

where $a=c_{d} g^{2}$ [2] and $c_{d}$ is a factor arising from momentum integrals in loop diagrams: $c_{d}=S_{d} /(2 \pi)^{d}$ with $S_{d}=2 \pi^{d / 2} / \Gamma(d / 2)$, so, e.g., $c_{4}=1 /\left(16 \pi^{2}\right)$, etc. An equivalent beta function is

$$
\beta_{a}=\frac{d a}{d t}=2 a \sum_{\ell=1}^{\infty} b_{\ell} a^{\ell}
$$

The one-loop coefficient $b_{1}$ is independent of the scheme used for regularization, and (for mass-independent schemes) this is also true for the two-loop coefficient $b_{2}$

Published by the American Physical Society under the terms of the Creative Commons Attribution 4.0 International license. Further distribution of this work must maintain attribution to the author(s) and the published article's title, journal citation, and DOI. Funded by SCOAP ${ }^{3}$.
$[3,4]$. In contrast, the higher-loop coefficients $b_{\ell}$ with $\ell \geq 3$ are scheme dependent [5]. It was thus expected that, at least for sufficiently small coupling, as in the deep ultraviolet (UV) limit of quantum chromodynamics (QCD), it would be possible to carry out a scheme transformation that eliminates these terms and yields a beta function with only one- and two-loop terms [6]. Such a scheme has been called the 't Hooft scheme. References [7-10] calculated explicit formulas for the effect of scheme transformations on the coefficients of a beta function and used these to construct scheme transformations that remove terms at loop order $\ell \geq 3$ from the beta function. A set of necessary conditions for a scheme transformation to be physically admissible was given in $[7,8]$, and it was shown that although these conditions can easily be satisfied if one applies a scheme transformation in the vicinity of the origin in coupling constant space, as in applications to optimized schemes in perturbative quantum chromodynamics [11], they are not automatically satisfied and are a significant constraint on the application of scheme transformations, when investigating a zero of the beta function away from the origin. In an asymptotically free theory, such a zero would be an infrared fixed point (IRFP) of the renormalization group, while in an infrared-free theory, such a zero would be an ultraviolet fixed point (UVFP) of the RG. An example of an IRFP occurs in an asymptotically free nonAbelian gauge theory with sufficiently many massless fermions [12], while an (exactly solved) example of a UVFP occurs in the $\mathrm{O}(N)$ nonlinear $\sigma$ model in $d=2+\epsilon$ dimensions in the large- $N$ limit [13].

In this work we consider the situation in which a theory has a beta function (which is not identically zero) with vanishing one-loop coefficient, $b_{1}=0$. We prove that in this case it is not, in general, possible to construct and apply a scheme transformation, even in the vicinity of the origin, $a=0$, that removes the scheme-dependent terms $b_{\ell}$ with 
$\ell \geq 3$ in the beta function. We discuss implications for the study of zeros of the beta function in this type of theory.

\section{SCHEME TRANSFORMATIONS}

In this section we give a brief review of relevant methodology on scheme transformations that will be needed here. One may define a scheme transformation as a mapping relating $a$ and $a^{\prime}$ given by

$$
a=a^{\prime} f\left(a^{\prime}\right),
$$

where $f\left(a^{\prime}\right)$ is the scheme transformation function. If the theory is free, then its properties must remain the same under a scheme transformation, so $f(0)=1$. The function $f\left(a^{\prime}\right)$ is taken to have the power series expansion

$$
f\left(a^{\prime}\right)=1+\sum_{s=1}^{s_{\max }} k_{s}\left(a^{\prime}\right)^{s},
$$

where $s_{\max }$ may be finite or infinite. The corresponding Jacobian $J=d a / d a^{\prime}$ has the series expansion

$$
J=1+\sum_{s=1}^{s_{\max }}(s+1) k_{s}\left(a^{\prime}\right)^{s} .
$$

The beta function in the transformed scheme is

$$
\beta_{a^{\prime}} \equiv \frac{d a^{\prime}}{d t}=\frac{d a^{\prime}}{d a} \frac{d a}{d t}=J^{-1} \beta_{a}
$$

This beta function has the series expansion

$$
\beta_{a^{\prime}}=2 a^{\prime} \sum_{\ell=1}^{\infty} b_{\ell}^{\prime}\left(a^{\prime}\right)^{\ell}
$$

with a new set of coefficients $b_{\ell}^{\prime}$. As noted above, the oneloop and two-loop coefficients are left invariant by this scheme transformation, i.e., $b_{1}^{\prime}=b_{1}$ and $b_{2}^{\prime}=b_{2}[3,4]$. Reference [7] presented explicit expressions for the $b_{\ell}^{\prime}$ in terms of the $b_{\ell}$ and $k_{s}$ for loop order $3 \leq \ell \leq 5$ and Ref. [8] extended these up to $\ell=8$ inclusive. For $b_{3}^{\prime}$ and $b_{4}^{\prime}$ these expressions are [7]

$$
b_{3}^{\prime}=b_{3}+k_{1} b_{2}+\left(k_{1}^{2}-k_{2}\right) b_{1}
$$

and

$b_{4}^{\prime}=b_{4}+2 k_{1} b_{3}+k_{1}^{2} b_{2}+\left(-2 k_{1}^{3}+4 k_{1} k_{2}-2 k_{3}\right) b_{1}$.

For the reader's convenience, we list some of these expressions for $b_{\ell}^{\prime}$ with higher $\ell$ in the Appendix, from Refs. [7,8].
As noted above, one important application of the study of schemes and scheme transformations is to calculations of higher-order corrections in perturbative QCD scattering processes at high energies. In this application, one is interested in choosing a scheme such that higher-order terms are small, so that one can achieve as accurate as possible a description of experimental data at a given order in perturbation theory. A different type of application is to the investigation of a possible zero of the beta function away from the origin in coupling constant space.

As specified in $[7,8]$, in order for a scheme transformation to be physically acceptable, it must satisfy the following necessary conditions:

(i) $\mathrm{C}_{1}$ : the scheme transformation must map a real positive $a$ to a real positive $a^{\prime}$, since a map taking $a>0$ to $a^{\prime}=0$ would be singular, and a map taking $a>0$ to a negative or complex $a^{\prime}$ would violate the unitarity of the theory.

(ii) $\mathrm{C}_{2}$ : the scheme transformation should not map a value of $a$ for which perturbation theory may be reliable, to a value of $a^{\prime}$ that is so large that perturbation theory is unreliable.

(iii) $\mathrm{C}_{3}: J$ should not vanish in the region of $a$ and $a^{\prime}$ of interest, or else there would be a pole in Eq. (2.4).

(iv) $\mathrm{C}_{4}$ : The existence of an IR or UV zero of $\beta$ has physical significance and must therefore be scheme independent. Hence, a scheme transformation must satisfy the condition that $\beta_{a}$ has a zero away from the origin if and only if $\beta_{a^{\prime}}$ has a corresponding zero away from the origin.

Clearly, these conditions apply both for a given scheme transformation and its inverse.

Although $b_{1}$ is nonzero in $\mathrm{QCD}$, there are theories in which $b_{1}$ may be zero (without the beta function being identically zero). One example of a theory in which $b_{1}$ can vanish is a vectorial non-Abelian gauge theory with gauge group $G$ and $N_{f}$ Dirac fermions transforming according to a representation $R$ of $G$. The one-loop coefficient of the beta function is [14]

$$
b_{1}=-\frac{1}{3}\left(11 C_{A}-4 N_{f} T_{f}\right),
$$

and the two-loop coefficient is [15]

$$
b_{2}=-\frac{1}{3}\left[34 C_{A}^{2}-4\left(5 C_{A}+3 C_{f}\right) N_{f} T_{f}\right]
$$

where $C_{A}$ and $C_{f}=C_{2}(R)$ are the quadratic Casimir invariants of the adjoint representation and the fermion representation $R$, respectively, and $T_{f}=T(R)$ is the trace invariant of $R$ [16]. The coefficient $b_{1}$ vanishes if $N_{f}=N_{f, b 1 z}$, where 


$$
N_{f, b 1 z}=\frac{11 C_{A}}{4 T_{f}}
$$

If $N_{f}=N_{f, b 1 z}$, then $b_{2}=C_{A}\left(7 C_{A}+11 C_{f}\right)$, which is positive, so the theory with $N_{f}=N_{f, b 1 z}$ is IR-free. As an explicit example, one could take $G=\mathrm{SU}(2)$ and $R$ equal to the fundamental representation, so that $N_{f, b 1 z}=11$. That is, an $\mathrm{SU}(2)$ gauge theory with these 11 Dirac fermions has $b_{1}=0$. Examples can also be given of chiral gauge theories in which, for a special choice of gauge group and fermion content, $b_{1}=0$. In all of these cases, the choice of parameters that renders $b_{1}=0$ leaves a nonzero $b_{2}$.

Moreover, the vanishing of $b_{1}$ can occur in scalar field theories; a recent example is a scalar theory with a cubic self-interaction in $d=6$ dimensions in which the scalar transforms as a bi-adjoint representation of a global $\mathrm{SU}(N) \otimes \mathrm{SU}(N)$ symmetry group [17]. In this theory, the first nonzero term in the beta function is $b_{2}$, which is negative. A study of a possible IRFP in this theory was carried out in [18].

A different type of situation occurs in an $\mathcal{N}=2$ supersymmetric gauge theory with gauge group $\mathrm{SU}\left(N_{c}\right)$ and $N_{f}$ matter hypermultiplets. A closed form expression for the beta function was calculated in [19] (see also [20]). This beta function has the property that $b_{n}=0$ if $n \geq 2$. By choosing $N_{f}$ appropriately, one can make $b_{1}=0$, so that the beta function vanishes identically. In contrast, here we discuss theories in which $b_{1}=0$ (either because of a special choice of parameters, as in Eq. (2.10), or identically, as in $[17,18])$, but the beta function is not identically zero.

\section{SCHEMES TO REMOVE TERMS IN BETA FUNCTION OF ORDER THREE LOOPS AND HIGHER}

An important application of scheme transformations is to the analysis of possible zero(s) of the beta function away from the origin. The beta function of an asymptotically free non-Abelian gauge theory has an ultraviolet zero at $a=\alpha /(4 \pi)=0$, which is a UVFP. If the theory contains sufficiently many massless fermions, the (perturbatively calculated) beta function may also have an infrared zero at a nonzero value of the gauge coupling. The theory is weakly coupled at this IRFP if the number of fermions is close to the upper limit allowed by asymptotic freedom, namely, $N_{f, b 1 z}$ in a theory with fermions in a single representation $R$, and hence is amenable to a perturbative treatment using series expansions in the variable $\Delta_{f}=N_{u}-N_{f}$ [12].

Since the terms of loop order $\ell \geq 3$ in the beta function are scheme dependent, so is the value of the IR zero when calculated to three-loop or higher-loop order. In order to understand the physical implications of this IR zero, it is necessary to assess the effect of scheme dependence on its value. A study of this dependence was carried out in [8,9] using several scheme transformations. Related studies were performed in [21-23].

One type of procedure that would be natural for a quantitative study of scheme dependence of a zero of the beta function would be to construct and apply a scheme transformation that would remove successively higher-loop terms in the beta function and, at each stage, determine how this removal shifted the position of the IR zero. Extending the results of [8], Ref. [9] constructed a set of scheme transformations $S_{R, m}$ with $m \geq 2$ with $k_{1}=0$ in Eq. (2.2) that remove the terms in the beta function at loop order $\ell=3$ to $\ell=m+1$, inclusive, and determined the range of $\alpha$ over which $S_{R, 2}$ and $S_{R, 3}$ could be applied to study the IR zero of the beta function of an asymptotically free gauge theory while satisfying the criteria to avoid introducing unphysical pathologies. Reference [10] presented a generalized one-parameter class of scheme transformation, denoted $S_{R, m, k_{1}}$ with $m \geq 2$, depending on $k_{1}$, with the property that an $S_{R, m, k_{1}}$ scheme transformation eliminates the $\ell$-loop terms in the beta function of a quantum field theory from loop order $\ell=3$ to order $\ell=m+1$, inclusive. A transformation in this class reduces to $S_{R, m}$ if $k_{1}=0$. These types of scheme transformations have also been used in the analysis of a possible ultraviolet zero in the beta functions of a $\mathrm{U}(1)$ gauge theory [24] and an $\mathrm{O}(N)$ $\lambda|\vec{\phi}|^{4}$ theory [25].

Although our focus here is on scheme transformations, we note that one can also analyze properties of (physical, gauge-invariant) operators at the IRFP in a non-Abelian gauge theory with sufficiently many fermions via series expansions in powers of $\Delta_{f}$. These have the advantage that they are manifestly scheme independent. This program has been carried out up to the $O\left(\left(\Delta_{f}\right)^{3}\right)$ level in [26,27] and up to the $O\left(\left(\Delta_{f}\right)^{4}\right)$ level in $[28,29]$, the latter using the fiveloop beta function [30,31].

To set the stage for our new results, we briefly recall the procedure for the construction of the $S_{R, m, k_{1}}$ scheme transformation in [10]. The first step is to use Eq. (2.6) and solve the equation $b_{3}^{\prime}=0$ for $k_{2}$. This yields the result

$k_{2}=\frac{b_{3}}{b_{1}}+\frac{b_{2}}{b_{1}} k_{1}+k_{1}^{2} \quad$ for $S_{R, m, k_{1}} \quad$ with $\quad m \geq 2$.

This suffices for $S_{R, 2, k_{1}}$. The reason that we solve for $k_{2}$ instead of $k_{1}$ is that this involves the solution of a linear equation for $k_{1}$, whereas the equation $b_{3}^{\prime}=0$ is a quadratic equation in $k_{1}$, so one would have to choose which of the two solutions of this quadratic would be used.

To obtain $S_{R, m, k_{1}}$ with $m \geq 3$, removing the $\ell=3,4$ terms in $\beta_{a^{\prime}}$, one substitutes the solution for $k_{2}$ from Eq. (3.1) into Eq. (2.7) and solves the equation $b_{4}^{\prime}=0$ for $k_{3}$. Again, this is a linear equation, with a unique solution, which is 


$$
\begin{aligned}
k_{3}= & \frac{b_{4}}{2 b_{1}}+\frac{3 b_{3}}{b_{1}} k_{1}+\frac{5 b_{2}}{2 b_{1}} k_{1}^{2}+k_{1}^{3} \text { for } S_{R, m, k_{1}} \\
& \text { with } \quad m \geq 3 .
\end{aligned}
$$

One continues in this manner to determine the $k_{s}$ with $s \geq 4$ such as to remove the terms in the beta function up to successively higher loop orders.

This procedure requires $b_{1}$ to be nonzero, since otherwise various multiplicative factors involving $1 / b_{1}$ in the $k_{s}$ with $s \geq 2$ and higher powers of $1 / b_{1}$ in the $k_{s}$ with $s \geq 4$ would be singular. Here we investigate the situation where $b_{1}=0$.

\section{IMPOSSIBILITY OF REMOVING ALL HIGHER-LOOP TERMS WITH $\ell \geq 3$ IF $b_{1}=0$ : CASE WHERE $b_{2} \neq 0$}

In this and the next section we show that if the one-loop term in the beta function is zero, i.e., if $b_{1}=0$, then it is, in general, not possible to apply Eqs. (2.1) and (2.2) to transform to a scheme in which all of the scheme-dependent $\ell$-loop coefficients with $\ell \geq 3$ are zero. We begin in this section with the case where $b_{1}=0$ and $b_{2} \neq 0$. If $b_{2}>0$, then this theory is IR-free, while if $b_{2}<0$, the theory is UV-free (i.e., asymptotically free).

We proceed to analyze scheme transformations intended to try to set higher-loop coefficients equal to zero. From Eq. (2.6), it follows that in order to have $b_{3}^{\prime}=0$, the unique solution for $k_{1}$ in the scheme transformation (2.1) and (2.2) is

$$
k_{1}=-\frac{b_{3}}{b_{2}}
$$

Substituting this in Eq. (2.7) for $b_{4}^{\prime}$, we obtain

$$
b_{4}^{\prime}=b_{4}-\frac{b_{3}^{2}}{b_{2}} .
$$

In general, this is nonzero. This proves that if $b_{1}=0$, then there is, in general, no scheme transformation of the form (2.1) with (2.2) that can be used to render $b_{4}^{\prime}$ zero, and hence, a fortiori, it is not possible to remove all of the scheme-dependent terms in the beta function.

For completeness, we comment on the extent to which one can remove higher-loop terms with $\ell \geq 5$ in this case. Substituting the value of $k_{1}$ from (4.1) in Eq. (A1) for $b_{5}^{\prime}$, we obtain

$$
b_{5}^{\prime}=b_{5}-\frac{3 b_{3} b_{4}}{b_{2}}+\frac{3 b_{3}^{3}}{b_{2}^{2}}-2 k_{2} b_{3}-k_{3} b_{2} .
$$

It is always possible to render this $b_{5}^{\prime}=0$ by setting

$$
k_{3}=\frac{b_{5}}{b_{2}}-\frac{3 b_{3} b_{4}}{b_{2}^{2}}+\frac{3 b_{3}^{3}}{b_{2}^{3}}-\frac{2 k_{2} b_{3}}{b_{2}} .
$$

As is evident from Eq. (4.4), there is thus an infinite set of pairs $\left(k_{2}, k_{3}\right)$ that render $b_{5}^{\prime}=0$. Note that in the special case where $b_{3}=0, k_{3}$ takes on the unique value $k_{3}=$ $b_{5} / b_{2}$ while $k_{2}$ is undetermined. Examining Eq. (A2), we see that with $k_{3}$ given by Eq. (4.4), one can solve the equation $b_{6}^{\prime}=0$ (as a linear equation in $k_{4}$ ) for a value of $k_{4}$. Similarly, with these $k_{s}$ values chosen, one can solve $b_{7}^{\prime}=0$ (as a linear equation in $k_{5}$ ) for $k_{5}$, and so forth for higher $b_{\ell}^{\prime}$.

Thus, in the transformed scheme,

$$
\begin{aligned}
\beta_{a^{\prime}} & =2\left(a^{\prime}\right)^{3}\left[b_{2}+b_{4}^{\prime}\left(a^{\prime}\right)^{2}\right] \\
& =2\left(a^{\prime}\right)^{3}\left[b_{2}+\left(b_{4}-\frac{b_{3}^{2}}{b_{2}}\right)\left(a^{\prime}\right)^{2}\right] .
\end{aligned}
$$

The function $\beta_{a^{\prime}}$ has a formal zero away from the origin at $a=a_{z}$, where

$$
a_{z}^{\prime}=\left[-\frac{b_{2}}{b_{4}-\frac{b_{3}^{2}}{b_{2}}}\right]^{1 / 2} .
$$

This is physical if the expression in the square root is positive. There are two cases with nonzero $b_{2}$ to consider, namely, $b_{2}<0$ and $b_{2}>0$. Let us first consider the case $b_{2}<0$, where the theory is UV-free. Then the condition that $a_{z}^{\prime}$ is physical is that $b_{4}-\left(b_{3}^{2} / b_{2}\right)>0$, i.e.,

$b_{4}>-\frac{b_{3}^{2}}{\left|b_{2}\right|} \quad$ for IRFP if $b_{1}=0 \quad$ and $\quad b_{2}<0$.

This is a necessary condition for the theory to have a physical IRFP at $a_{z}^{\prime}$, but is not sufficient; the scheme transformation to the primed scheme must also satisfy the conditions $\mathrm{C}_{1}-\mathrm{C}_{4}$ from $[7,8]$ listed above.

Next, we consider the case $b_{2}>0$, where the theory is IR-free. Here the condition that $a_{z}^{\prime}>0$ is that $b_{4}-\left(b_{3}^{2} / b_{2}\right)<0$, i.e.,

$$
b_{4}<\frac{b_{3}^{2}}{b_{2}} \quad \text { for UVFP if } b_{1}=0 \text { and } \quad b_{2}>0 .
$$

Again, this is a necessary but not sufficient condition for the theory to a physical UVFP at $a_{z}^{\prime}$; the scheme transformation must also satisfy conditions $\mathrm{C}_{1}-\mathrm{C}_{4}$.

\section{IMPOSSIBILITY OF REMOVING ALL HIGHER-LOOP TERMS WITH $\ell \geq 3$ IF $b_{1}=0$ : CASE WHERE $b_{2}=0$}

Next, we consider the case in which both $b_{1}=0$ and $b_{2}=0$, i.e., the maximal scheme-independent part of the beta function is zero. Here, Eq. (2.6) reduces to 


$$
b_{3}^{\prime}=b_{3} \text {. }
$$

In the generic situation in which $b_{3} \neq 0$, this immediately proves that if $b_{1}=b_{2}=0$, then there is, in general, no scheme transformation that one can use to remove all higherloop terms with $\ell \geq 3$ in the beta function. Here we assume that $b_{3} \neq 0$ and comment on the special case $b_{3}=0$ below. We remark on specific results for other higher-loop coefficients. The condition that $b_{4}^{\prime}=0$ can be satisfied by choosing

$$
k_{1}=-\frac{b_{4}}{2 b_{3}}
$$

Substituting this into Eq. (A1), we see that it is possible to render $b_{5}^{\prime}=0$ with the choice

$$
k_{2}=-\frac{b_{5}}{b_{3}}+\frac{b_{4}^{2}}{b_{3}^{2}}
$$

Substituting these values of $k_{1}$ and $k_{2}$ into the expression (A2), we obtain

$$
b_{6}^{\prime}=b_{6}-\frac{2 b_{4} b_{5}}{b_{3}}+\frac{b_{4}^{3}}{b_{3}^{2}} .
$$

This does not contain dependence on any other $k_{s}$ that can be chosen to make it zero, and, in general, it is nonzero. With $k_{1}$ and $k_{2}$ set equal to their values in Eqs. (5.2) and (5.3), there is an infinite number of values of the pair $\left(k_{3}, k_{4}\right)$ that can be used to render $b_{7}^{\prime}=0$. Having done this, one can choose $k_{5}$ to make $b_{8}^{\prime}=0$. Higher-loop coefficients can be analyzed in a similar manner.

We next consider the hypothetical case in which not only $b_{1}=0$ and $b_{2}=0$, but also one starts in a scheme in which some finite number of higher-loop coefficients $b_{\ell}$ with $3 \leq$ $\ell \leq p$ are zero. As before, we find that it is not, in general, possible to construct a scheme transformation that renders all of the $b_{\ell}$ with $\ell \geq p+1$ zero. This is simply proved by noting that our general results in Refs. $[7,8]$ have the form

$$
b_{\ell}^{\prime}=b_{\ell}+(\ell-2) k_{1} b_{\ell-1}+\cdots \quad \text { for } \ell \geq 3,
$$

where the $\cdots$ in Eq. (5.5) denote a sum of $k_{s}$-dependent coefficients times the coefficients $b_{k}$ with $1 \leq k \leq \ell-2$. Hence, if $b_{\ell}=0$ for $1 \leq \ell \leq p$, then $b_{p+1}^{\prime}=b_{p+1}$. Since, by assumption, $b_{p+1}$ is nonzero, so is $b_{p+1}^{\prime}$, which proves our claim.

\section{CONCLUSIONS}

In conclusion, in this work we have proved that if the beta function of a theory is not identically zero and if the one-loop term in this beta function is zero, then, in general, it is not possible to transform to a scheme where all of the schemedependent coefficients $b_{\ell}^{\prime}$ with $\ell \geq 3$ are zero. We have given explicit results for several specific cases, including the case in which $b_{1}=0$ but $b_{2} \neq 0$ and the case where $b_{1}=b_{2}=0$. In the case of greatest physical interest, namely, where $b_{1}=0$ and $b_{2} \neq 0$, we have also discussed resultant necessary (but not sufficient) conditions for the existence of a zero in the beta function away from the origin.

\section{ACKNOWLEDGMENTS}

We thank J. Gracey for valuable discussions in connection with [17] and collaboration on [18]. This research was supported in part by the U.S. National Science Foundation Grant No. NSF-PHY-1915093 (R. S.).

\section{APPENDIX: EXPRESSIONS FOR THE BETA FUNCTION COEFFICIENTS $b_{e}^{\prime}$}

For reference, we list some of the higher-loop coefficients $b_{\ell}^{\prime}$ with $\ell \geq 5$ from Refs. [7,8]:

$$
\begin{aligned}
b_{5}^{\prime}=b_{5}+ & 3 k_{1} b_{4}+\left(2 k_{1}^{2}+k_{2}\right) b_{3}+\left(-k_{1}^{3}+3 k_{1} k_{2}-k_{3}\right) b_{2}+\left(4 k_{1}^{4}-11 k_{1}^{2} k_{2}+6 k_{1} k_{3}+4 k_{2}^{2}-3 k_{4}\right) b_{1}, \\
b_{6}^{\prime}= & b_{6}+4 k_{1} b_{5}+\left(4 k_{1}^{2}+2 k_{2}\right) b_{4}+4 k_{1} k_{2} b_{3}+\left(2 k_{1}^{4}-6 k_{1}^{2} k_{2}+4 k_{1} k_{3}+3 k_{2}^{2}-2 k_{4}\right) b_{2} \\
& +\left(-8 k_{1}^{5}+28 k_{1}^{3} k_{2}-16 k_{1}^{2} k_{3}-20 k_{1} k_{2}^{2}+8 k_{1} k_{4}+12 k_{2} k_{3}-4 k_{5}\right) b_{1},
\end{aligned}
$$

and

$$
\begin{aligned}
b_{7}^{\prime}= & b_{7}+5 k_{1} b_{6}+\left(7 k_{1}^{2}+3 k_{2}\right) b_{5}+\left(2 k_{1}^{3}+7 k_{1} k_{2}+k_{3}\right) b_{4}+\left(k_{1}^{4}-2 k_{1}^{2} k_{2}+4 k_{1} k_{3}+3 k_{2}^{2}-k_{4}\right) b_{3} \\
& +\left(-4 k_{1}^{5}+15 k_{1}^{3} k_{2}-9 k_{1}^{2} k_{3}-12 k_{1} k_{2}^{2}+9 k_{2} k_{3}+5 k_{1} k_{4}-3 k_{5}\right) b_{2} \\
& +\left(16 k_{1}^{6}-68 k_{1}^{4} k_{2}+40 k_{1}^{3} k_{3}-21 k_{1}^{2} k_{4}+73 k_{1}^{2} k_{2}^{2}-58 k_{1} k_{2} k_{3}+10 k_{1} k_{5}+16 k_{2} k_{4}-12 k_{2}^{3}+9 k_{3}^{2}-5 k_{6}\right) b_{1} .
\end{aligned}
$$


[1] C. G. Callan, Phys. Rev. D 2, 1541 (1970); K. Symanzik, Commun. Math. Phys. 18, 227 (1970).

[2] For a scalar theory in $d=4$ dimensions with a $\lambda \phi^{4}$ interaction, Eq. (1.1) would be $\beta_{\lambda}=\lambda \sum_{\ell=1}^{\infty} b_{\ell} a^{\ell}$ with $a=\lambda /\left(16 \pi^{2}\right)$. Our conclusions apply to all of these theories, but we use notation specific to the $d=4$ gauge theory and $d=6$ cubic scalar theory.

[3] E. Brézin, J. Le Guillou, and J. Zinn-Justin, Phys. Rev. D 9 , 1121 (1974).

[4] D. J. Gross, in Methods in Field Theory, edited by R. Balian and J. Zinn-Justin (Les Houches, North-Holland, Amsterdam, 1976), p. 141.

[5] A commonly used scheme is the $\overline{\mathrm{MS}}$ scheme, presented in W. A. Bardeen, A. J. Buras, D. W. Duke, and T. Muta, Phys. Rev. D 18, 3998 (1978); see also G. 't Hooft, Nucl. Phys. B61, 455 (1973).

[6] G. 't Hooft, in The Whys of Subnuclear Physics, edited by A. Zichichi, Proceedings of the 1977 Erice Summer School (Plenum, New York, 1979), p. 943.

[7] T. A. Ryttov and R. Shrock, Phys. Rev. D 86, 065032 (2012).

[8] T. A. Ryttov and R. Shrock, Phys. Rev. D 86, 085005 (2012).

[9] R. Shrock, Phys. Rev. D 88, 036003 (2013).

[10] R. Shrock, Phys. Rev. D 90, 045011 (2014).

[11] See S. J. Brodsky and X.-G. Wu, Phys. Rev. Lett. 109, 042002 (2012); M. Mojaza, S. J. Brodsky, and X.-G. Wu, Phys. Rev. Lett. 110, 192001 (2013), and references therein to earlier work.

[12] T. Banks and A. Zaks, Nucl. Phys. B196, 189 (1982).

[13] W. A. Bardeen, B. W. Lee, and R. E. Shrock, Phys. Rev. D 14, 985 (1976); E. Brézin and J. Zinn-Justin, Phys. Rev. B 14, 3110 (1976); see also A. Polyakov, Phys. Lett. 59B, 79 (1975).

[14] D. J. Gross and F. Wilczek, Phys. Rev. Lett. 30, 1343 (1973); H. D. Politzer, Phys. Rev. Lett. 30, 1346 (1973); G. 't Hooft (unpublished).

[15] W. E. Caswell, Phys. Rev. Lett. 33, 244 (1974); D. R. T. Jones, Nucl. Phys. B75, 531 (1974).
[16] We recall the general definitions of these group invariants. Denote $T_{R}$ as a generator of the Lie algebra of a group $G$ in the representation $R$. Then the quadratic Casimir invariant $C_{2}(R)$ is defined by $T_{R}^{a} T_{R}^{a}=C_{2}(R) I$, where $I$ is the $d_{R} \times d_{R}$ identity matrix, and the trace invariant $T(R)$ is defined by $\operatorname{Tr}_{R}\left(T_{R}^{a} T_{R}^{b}\right)=T(R) \delta^{a b}$, where $1 \leq a \leq o(G)$, with $o(G)$ the order of the group. We write $C_{A}=C_{2}(\mathrm{Adj})$ and $C_{f}=C_{2}\left(R_{f}\right)$. For $\mathrm{SU}\left(N_{c}\right), C_{2}(\mathrm{Adj})=T(\mathrm{Adj})=N_{c}$ and for the fundamental representation, $C_{f}=\left(N_{c}^{2}-1\right) /\left(2 N_{c}\right)$ and $T_{f}=1 / 2$.

[17] J. A. Gracey, Phys. Rev. D 101, 125022 (2020).

[18] J. A. Gracey, T. A. Ryttov, and R. Shrock, Phys. Rev. D 102, 045016 (2020).

[19] V. A. Novikov, M. A. Shifman, A. J. Vainshtein, and V. I. Zakharov, Nucl. Phys. B229, 381 (1983).

[20] See also M. A. Shifman and K. V. Stepanyantz, Phys. Rev. D 91, 105008 (2015), and references therein.

[21] T. Ryttov, Phys. Rev. D 89, 016013 (2014); 89, 056001 (2014); 90, 056007 (2014).

[22] G. Choi and R. Shrock, Phys. Rev. D 90, 125029 (2014).

[23] J. A. Gracey and R. M. Simms, Phys. Rev. D 91, 085037 (2015).

[24] R. Shrock, Phys. Rev. D 89, 045019 (2014).

[25] R. Shrock, Phys. Rev. D 90, 065023 (2014); 96, 056010 (2017).

[26] T. A. Ryttov, Phys. Rev. Lett. 117, 071601 (2016).

[27] T. A. Ryttov and R. Shrock, Phys. Rev. D 94, 125005 (2016).

[28] T. A. Ryttov and R. Shrock, Phys. Rev. D 94, 105014 (2016).

[29] T. A. Ryttov and R. Shrock, Phys. Rev. D 95, 105004 (2017); 96, 105015 (2017); 97, 025004 (2018).

[30] P. A. Baikov, K. G. Chetyrkin, and J. H. Kühn, Phys. Rev. Lett. 118, 082002 (2017).

[31] F. Herzog, B. Ruijl, T. Ueda, J. A. M. Vermaseren, and A. Vogt, J. High Energy Phys. 02 (2017) 090. 